\title{
ON OPTIMIZING NONLINEAR ADAPTIVE CONTROL ALLOCATION WITH ACTUATOR DYNAMICS
}

\author{
Johannes Tjønnås and Tor A. Johansen
}

\author{
Department of Engineering Cybernetics, Norwegian University \\ of Science and Technology, Trondheim, Norway
}

\begin{abstract}
In this work we address the optimizing control allocation problem for a nonlinear over-actuated time-varying system where parameters affine in the dynamic actuator and effector model may be assumed unknown. In-stead of finding the optimal control allocation at each time instant, a dynamic approach is considered by constructing update-laws that represent asymptotically optimal allocation search and adaptation. Using Lyapunov analysis for cascaded set-stable systems, uniform global/local asymptotic stability is guaranteed for the optimal set described by the system, the optimal allocation update-law and the adaptive update-law. Simulations of a scaled-model ship, manoeuvred at low-speed, demonstrate the performance of the proposed allocation scheme.
\end{abstract}

Keywords: Control allocation; Adaptive control; Nonlinear systems; Cascaded systems.

\section{INTRODUCTION}

Consider the system dynamics

$$
\dot{x}=f_{x}(t, x)+g_{x}(t, x) \tau
$$

the effector model

$$
\tau:=\Phi(t, x, u, \theta):=\Phi_{0}(t, x, u)+\Phi_{\theta}(t, x, u) \theta
$$

and the actuator dynamics

$$
\dot{u}=f_{u 0}\left(t, x, u, u_{c m d}\right)+f_{u \theta}\left(t, x, u, u_{c m d}\right) \theta
$$

where $t \geq 0, x \in \mathbb{R}^{n}, u \in \mathbb{R}^{r}, \tau \in \mathbb{R}^{d}, \theta \in \mathbb{R}^{m}$ and $u_{c m d} \in \mathbb{R}^{c}$. The constant parameter vector $\theta$ contains parameters of the actuator and effector model, that will be viewed as uncertain parameters to be adapted in the low level control algorithm and used in the allocation scheme.

This work is motivated by the over-actuated control allocation problem $(d \leq r)$, where the problem is described by a nonlinear system, divided into high- (1) and low-level (3) dynamics and a static interconnection (2) of the two. The main contribution of this work is to show that the static optimal control allocation problem:

$$
\min _{u_{d}} J\left(t, x, u_{d}\right) \quad \text { s.t } \quad \tau_{x}-\Phi(t, x, u, \hat{\theta})=0,
$$

where $\hat{\theta} \in \mathbb{R}^{m}$ is an estimate of $\theta$, not necessarily needs to be solved exactly at each time instant.

Optimizing control allocation solutions have been derived for certain classes of over-actuated systems, such as aircraft, automotive vehicles and marine vessels, (Enns 1998, Sørdalen 1997, Bodson 2002, Luo et al. 2004, Luo et al. 2005, Poonamallee et al. 2005) and (Johansen et al. 2004). The control allocation problem is, in (Enns 1998, Sørdalen 1997) and (Bodson 2002), viewed as a static or quasi-dynamic problem that is solved independently of the dynamic control problem considering non-adaptive linear effector models of the form $\tau=G u$, neglecting the effect of actuator dynamics. In (Luo et al. 2004) and (Luo et al. 2005) a dynamic model predictive approach is considered to solve the allocation problem with linear time-varying dynamics in the actuator model, $T \dot{u}+u=u_{c m d}$. In (Poonamallee et al. 2005) and (Johansen et al. 2004) sequential quadratic programming techniques are used to cope with nonlinearities in the control allocation problem due to singularity avoidance.

The main advantage of the control allocation approach is in general modularity and the ability to handle redundancy and constraints. In the present work we consider dynamic solutions based on the ideas presented in (Johansen 2004) and (Tjønnås and Johansen 2005). In (Johansen 2004) it was shown that it is not necessary to solve the optimization problem (4) exactly at each time instant. Furthermore a control Lyapunov function was used to derive an exponentially convergent update-law for $u$ (related to a gradient or Newton-like optimization) such that the control allocation problem (4) could be solved dynamically. It was also shown that convergence and asymptotic optimality of the system, composed by the dynamic control allocation and a uniform globally exponen- 
tially stable trajectory-tracking controller, guarantees uniform boundedness and uniform global exponential convergence to the optimal solution of the system. In (Tjønnås and Johansen 2005) the results from (Johansen 2004) were extended by allowing uncertain parameters, associated with an adaptive law, in the effector model, and by applying set-stability analysis in order to also conclude asymptotic stability of the optimal solution.

In what follows we will extend the ideas from (Tjønnås and Johansen 2005) by introducing dynamics in the actuator model, and finally we present an example of this control allocation approach, where dynamic positioning (DP) of a model ship in different/changing weather conditions, controlled by thrusters experiencing thrust losses, is considered.

Whenever referring to the notion of set-stability, the set has the property of being nonempty, and we strictly follow the definitions given in (Tjønnås et al. 2006) motivated by (Teel et al. 2002) and (Lin et al. 1996).

\section{ADAPTIVE CONTROL ALLOCATION WITH ACTUATOR DYNAMICS}

Our results rests on the following assumptions:

\section{Assumption 1. (Plant)}

a) The states of (1) and (3) are know for all $t>t_{0}$.

b) There exist a continuous function $G_{f}: \mathbb{R}_{>0} \rightarrow$ $\mathbb{R}_{>0}$ and a constant $B_{g}$, such that $\left|f_{x}(t, \bar{x})\right| \leq$ $G_{f}(|x|)$ and $\left|g_{x}(t, x)\right| \leq B_{g}$ for all $t$ and $x$. Moreover $f_{x}$ is locally Lipschitz in $t$ and $x$.

c) There exist a continuous function $\varsigma_{\partial g_{x}}: \mathbb{R}_{\geq 0} \rightarrow$ $\mathbb{R}_{\geq 0}$ such that for all $t$ and $x, g_{x}$ is differentiable and $\left|\frac{\partial g_{x}}{\partial t}\right|+\left|\frac{\partial g_{x}}{\partial x}\right| \leq \varsigma_{\partial g_{x}}(|x|)$.

d) The function $\Phi$ from the static mapping (2) is twice differentiable and there exist a continuous function $G_{\Phi}: \mathbb{R}_{\geq 0} \rightarrow \mathbb{R}_{\geq 0}$, such that $\left|\Phi_{\theta}\right|+\left|\Phi_{0}\right| \leq$ $G_{\Phi}\left(\left|\left(x^{\mathrm{T}}, u^{\mathrm{T}}\right)^{\mathrm{T}}\right|\right)$ for all $t, x$, and $u$. Further there exist a continuous function $\varsigma_{\partial \Phi}(|x|) \geq\left|\frac{\partial \Phi_{\theta}}{\partial t}\right|+$ $\left|\frac{\partial \Phi_{0}}{\partial t}\right|+\left|\frac{\partial \Phi_{\theta}}{\partial x}\right|+\left|\frac{\partial \Phi_{0}}{\partial x}\right|+\left|\frac{\partial \Phi_{\theta}}{\partial u}\right|+\left|\frac{\partial \Phi_{0}}{\partial u}\right|$ where $\varsigma_{\partial \Phi}: \mathbb{R}_{>0} \rightarrow \mathbb{R}_{>0}$.

e) There exists constants $\varrho_{2}>\varrho_{1}>0$, such that $\forall t, x, u$ and $\theta$

$$
\varrho_{1} I \leq \frac{\partial \Phi}{\partial u}(t, x, u, \theta)\left(\frac{\partial \Phi}{\partial u}(t, x, u, \theta)\right)^{\mathrm{T}} \leq \varrho_{2} I .
$$

Assumption 2. (High/low level control algorithms)

a) There exists a high level control $\tau_{x}:=k_{x}(t, x)$, where $\left|k_{x}(t, x)\right| \leq \varsigma_{k}(|x|)$ and $\varsigma_{k}: \mathbb{R}_{\geq 0} \mapsto \mathbb{R}_{\geq 0}$ is a continuous function, that render the equilibrium of (1) UGAS for $\tau=\tau_{x}$. The function $k_{x}$ is differentiable, $\left|\frac{\partial k_{x}}{\partial t}\right|+\left|\frac{\partial k_{x}}{\partial x}\right| \leq \varsigma_{\partial k_{x}}(|x|)$, where $\varsigma_{\partial k_{x}}: \mathbb{R}_{>0} \mapsto \mathbb{R}_{>0}$ is continuous.

b) There exists a low-level control

$u_{c m d}:=k_{u}\left(t, x, u, u_{d}, \dot{u}_{d}, \hat{\theta}\right)$ that makes the equilibrium of

$$
\dot{\tilde{u}}=f_{\tilde{u}}\left(t, x, \tilde{u}, u_{d}, \hat{\theta}, \theta\right),
$$

where $\tilde{u}:=u-u_{d}$ and $f_{\tilde{u}}\left(t, x, \tilde{u}, u_{d}, \hat{\theta}, \theta\right):=$ $f_{u 0}\left(t, x, u, u_{c m d}\right)+f_{u \theta}\left(t, x, u, u_{c m d}\right) \theta-f_{d}\left(t, x, \tilde{u}, u_{d}, \hat{\theta}\right)$, UGAS if $\hat{\theta}=\theta$, and if $x, u_{d}, \dot{u}_{d}$ exist for all $t>0$. Furthermore $k_{u}\left(t, x, u, u_{d}, \dot{u}_{d}, \hat{\theta}\right) \leq \varsigma_{u}\left(\left|u-u_{d}\right|\right)$, where $\varsigma_{u}: \mathbb{R}_{\geq 0} \mapsto \mathbb{R}_{\geq 0}$, is a continuous differentiable function. Moreover $\left|\frac{\partial k_{u}}{\partial t}\right|+\left|\frac{\partial k_{u}}{\partial x}\right|+$ $\left|\frac{\partial k_{u}}{\partial u}\right|+\left|\frac{\partial k_{u}}{\partial u_{d}}\right|+\left|\frac{\partial k_{u}}{\partial \dot{u}_{d}}\right| \leq \varsigma \partial k_{u}\left(\left|u-u_{d}\right|\right)$, where

$\varsigma_{\partial k_{u}}: \mathbb{R}_{\geq 0} \mapsto \mathbb{R}_{\geq 0}$ is continuous.

Remark 1. From assumption $2 \mathrm{a}$ ) there exist a Lyapunov function $V_{x}: \mathbb{R}_{\geq 0} \times \mathbb{R}^{n} \mapsto \mathbb{R}_{\geq 0}$ that satisfy:

$$
\begin{aligned}
\alpha_{x 1}(|x|) & \leq V_{x}(t, x) \leq \alpha_{x 2}(|x|) \\
\frac{\partial V_{x}}{\partial t}+\frac{\partial V_{x}}{\partial x} f(t, x) & \leq-\alpha_{x 3}(|x|) \\
\left|\frac{\partial V_{x}}{\partial x}\right| & \leq \alpha_{x 4}(|x|),
\end{aligned}
$$

where $\alpha_{x 1}, \alpha_{x 2}, \alpha_{x 3}, \alpha_{x 4} \in \mathcal{K}_{\infty}$, for the system $\dot{x}=f(t, x):=f_{x}(t, x)+g_{x}(t, x) k_{x}(t, x)$, where $g(t, x):=g_{x}(t, x)$. If $x(t), u_{d}(t), \dot{u}_{d}(t)$, and $\hat{\theta}(t)=$ $\theta(t)$ exist for all $t$ then there also exist a function $V_{\tilde{u}}: \mathbb{R}_{\geq 0} \times \mathbb{R}^{n} \rightarrow \mathbb{R}_{\geq 0}$ and $\mathcal{K}_{\infty}$ functions $\alpha_{\tilde{u} 1}, \alpha_{\tilde{u} 2}$, $\alpha_{\tilde{u} 3}$ such that

$$
\begin{aligned}
\alpha_{\tilde{u} 1}(|\tilde{u}|) & \leq V_{\tilde{u}}(t, \tilde{u}) \leq \alpha_{\tilde{u} 2}(|\tilde{u}|) \\
\frac{\partial V_{\tilde{u}}}{\partial t}+\frac{\partial V_{\tilde{u}}}{\partial \tilde{u}} f_{\tilde{u}}\left(t, x(t), \tilde{u}, u_{d}(t), \hat{\theta}(t), \theta(t)\right) & \leq-\alpha_{\tilde{u} 3}(|\tilde{u}|) .
\end{aligned}
$$

We will not discuss the details in these assumptions, but they are sufficient in order to guarantee existence of solutions and validity of the update-laws that we propose in this paper.

By assumption 2 a) we may consider a Series Parallel estimation model:

$\dot{\hat{u}}=A_{\hat{u}}(u-\hat{u})+f_{u 0}\left(t, x, u, u_{c m d}\right)+f_{u \theta}\left(t, x, u, u_{c m d}\right) \hat{\theta}$,

where $\left(-A_{\hat{u}}\right)$ is Hurwitz. This estimation model is used in a Lyapunov based indirect scheme in order to estimate the unknown but bounded parameter vector $\theta$. For analytical purpose we use the error estimate:

$$
\dot{\eta}=-A_{\hat{u}} \eta+f_{u \theta}\left(t, x, u, u_{c m d}\right) \tilde{\theta}
$$

where $\eta:=u-\hat{u}$ and $\tilde{\theta}:=\theta-\hat{\theta}$.

The adaptive structure of the algorithm, that we will present, is based on defining the adaptive law at the low level, incorporating the low level control algorithm and passing these parameter estimates to the control allocation algorithm. Generally we consider an adaptive control allocation approach of four modular steps where the contribution of this paper is on step three and four and the interconnection of these steps, see also Figure 1.

(1) The high-level control algorithm. The virtual control $\tau$ is treated as an available input to the system (1), and a virtual control law $\tau_{x}$ is designed such that the origin of (1) is UGAS when $\tau=\tau_{x}$

(2) The low-level control algorithm. The actuator dynamic is controlled by the actuators through an control law $u_{c m d}$, such that for any smooth reference $u_{d}, u$ tracks $u_{d}$ asymptotically.

(3) The control allocation algorithm (Connecting the high and low level control). Based on the minimization problem (4) where $J$ is a cost function that incorporates objectives such as minimum power consumption and actuator constraints (implemented as barrier functions), the Lagrangian function

$$
\begin{aligned}
& L_{\hat{\theta}}\left(t, x, u_{d}, \tilde{u}, \lambda\right):=J\left(t, x, u_{d}\right) \\
& \quad+\left(k_{x}(t, x)-\Phi\left(t, x, u_{d}+\tilde{u}, \hat{\theta}\right)\right)^{\mathrm{T}} \lambda
\end{aligned}
$$



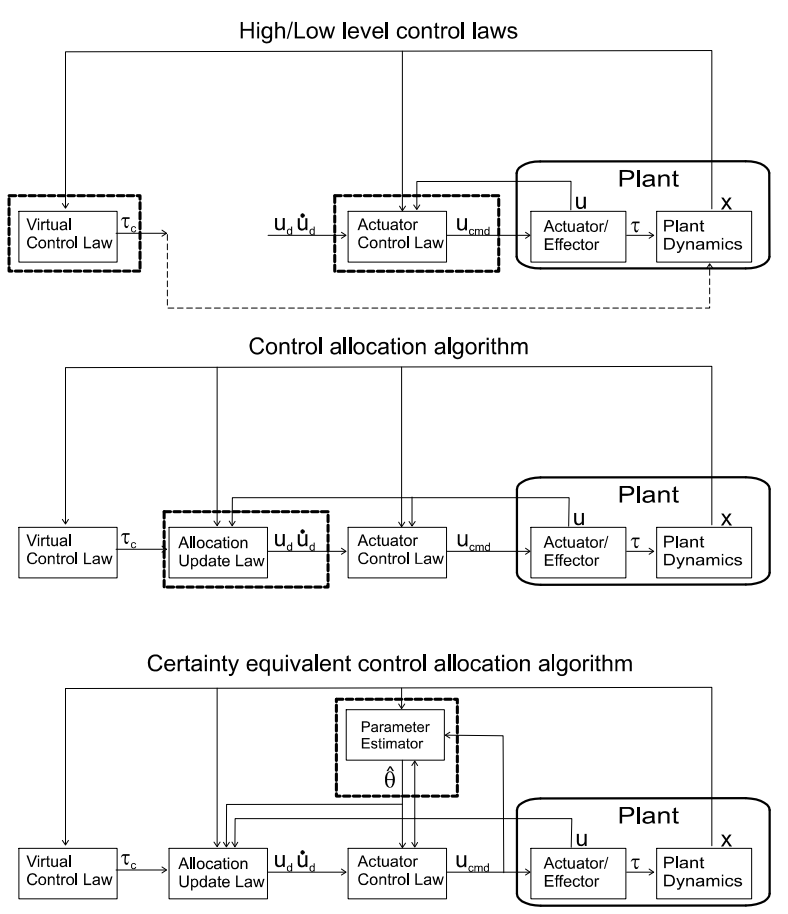

Fig. 1. Adaptive control allocation design philosophy

is introduced, and update laws for the effector reference $u_{d}$ and the Lagrangian parameter $\lambda$ are then defined such that $u_{d}$ and $\lambda$ converges to a set defined by the time-varying optimality condition.

(4) The adaptive algorithm. In order to cope with an possibly unknown parameter vector $\theta$ in the effector and actuator models, an adaptive law is defined. The parameter estimate is used in the control allocation scheme and a certainty equivalent adaptive optimal control allocation algorithm can be defined.

Based on Assumption 1 and 2 together with the following assumption;

Assumption 3. (Optimal control allocation)

a) The cost function $J: \mathbb{R}_{\geq t_{0}} \times \mathbb{R}^{n \times r} \rightarrow \mathbb{R}$ is twice differentiable and satisfies: $J\left(t, x, u_{d}\right) \rightarrow \infty$ as $\left|u_{d}\right| \rightarrow \infty$. Further there exists a continuous function $\varsigma_{\partial^{2} J}: \mathbb{R}_{\geq 0} \rightarrow \mathbb{R}_{\geq 0}$ such that $\left|\frac{\partial^{2} J}{\partial t \partial u_{d}}\right|+$ $\left|\frac{\partial^{2} J}{\partial x \partial u_{d}}\right| \leq \varsigma_{\partial^{2} J}\left(\left|\left(x^{\mathrm{T}}, u_{d}^{\mathrm{T}}, \lambda^{\mathrm{T}}\right)\right|\right) \forall t, x, u_{d}$ and $\lambda$.

b) There exists constants $k_{2}>k_{1}>0$, such that $\forall t$, $x, \tilde{u}, \hat{\theta}$ and $\left(u_{d}^{\mathrm{T}}, \lambda^{\mathrm{T}}\right)^{\mathrm{T}} \notin \mathcal{O}_{u_{d} \lambda}$, where

$\mathcal{O}_{u_{d} \lambda}(t, x):=\left\{\left(u_{d}^{\mathrm{T}}, \lambda^{\mathrm{T}}\right) \in \mathbb{R}^{r+d} \mid\left(\frac{\partial L_{\hat{\theta}}^{\mathrm{T}}}{\partial u_{d}}, \frac{\partial L_{\hat{\theta}}^{\mathrm{T}}}{\partial \lambda}\right)=0\right\}$, then

$$
k_{1} I \leq \frac{\partial^{2} L_{\hat{\theta}}}{\partial u_{d}^{2}}\left(t, x, u_{d}, \tilde{u}, \lambda, \hat{\theta}\right) \leq k_{2} I .
$$

If $\left(u_{d}^{\mathrm{T}}, \lambda^{\mathrm{T}}\right)^{\mathrm{T}} \in \mathcal{O}_{u_{d} \lambda}$, the lower bound is replaced by $\frac{\partial^{2} L_{\hat{\theta}}}{\partial u_{d}^{2}} \geq 0$.

we formulate our main problem:

Problem: Define update-laws for $u_{d}, \hat{\theta}$ and $\lambda$ such that the stability of the closed loop:

$$
\Sigma_{1}:\left\{\begin{array}{l}
\dot{x}=f(t, x) \\
+g(t, x)\left(\Phi\left(t, x, u_{d}+\tilde{u}, \theta\right)-k_{x}(t, x)\right)
\end{array}\right.
$$

$$
\Sigma_{2}:\left\{\begin{array}{l}
\dot{\tilde{u}}=f_{\tilde{u}}\left(t, x, \tilde{u}, u_{d}, \hat{\theta}, \theta\right) \\
\dot{u}_{d}=f_{d}\left(t, x, \tilde{u}, u_{d}, \hat{\theta}\right) \\
\dot{\lambda}=f_{\lambda}\left(t, x, u_{d}, \hat{\theta}\right) \\
\dot{\eta}=-A \eta+f_{u \theta}\left(t, x, u, u_{c m d}\right) \tilde{\theta} \\
\dot{\tilde{\theta}}=-f_{\hat{\theta}}\left(t, x, u_{c m d}, \tilde{u}, u_{d}, \hat{\theta}\right),
\end{array}\right.
$$

from (1), (2), (3), (6) and (13), is conserved and $u_{d}(t)$ converges to an optimal solution with respect to the minimization problem (4).

Note that system (16)-(17) takes the form of a cascade as long as $x(t)$ exists, and is viewed as a time-varying input to $\Sigma_{2}$, for all $t>0$. We deal with the problem formulated by: i) defining a Lyapunov like function, $V_{u_{d} \lambda \tilde{u} \eta \tilde{\theta}}$, for system $\Sigma_{2}$ and defining explicit updatelaws for $u_{d}, \lambda$ and $\tilde{\theta}$ such that $\dot{V}_{u_{d} \lambda \tilde{u} \eta \tilde{\theta}} \leq 0$. ii) Further, we prove boundedness of the closed-loop system, $\Sigma_{1}$ and $\Sigma_{2}$, and use the cascade lemma (Tjønnås et al. 2006) to prove convergence and stability.

Based on the Lyapunov like function candidate

$$
\begin{aligned}
& V_{u_{d} \lambda \tilde{u} \eta \tilde{\theta}}\left(t, x, u_{d}, \lambda, \tilde{u}, \eta\right):=V_{\tilde{u}}(t, \tilde{u})+\frac{1}{2} \eta^{\mathrm{T}} \Gamma_{\eta} \eta \\
& +\frac{1}{2}\left(\frac{\partial L_{\hat{\theta}}^{\mathrm{T}}}{\partial u_{d}} \frac{\partial L_{\hat{\theta}}}{\partial u_{d}}+\frac{\partial L_{\hat{\theta}}^{\mathrm{T}}}{\partial \lambda} \frac{\partial L_{\hat{\theta}}}{\partial \lambda}\right)+\frac{1}{2} \tilde{\theta}^{\mathrm{T}} \Gamma_{\tilde{\theta}} \tilde{\theta}
\end{aligned}
$$

we suggest the following control allocation algorithm:

$$
\begin{aligned}
& \left(\dot{u}_{d}^{\mathrm{T}}, \dot{\lambda}^{\mathrm{T}}\right)^{\mathrm{T}}=-\Gamma \mathbb{H}_{\hat{\theta}}\left(\frac{\partial L_{\hat{\theta}}^{\mathrm{T}}}{\partial u_{d}}, \frac{\partial L_{\hat{\theta}}^{\mathrm{T}}}{\partial \lambda}\right)^{\mathrm{T}}-u_{f f \hat{\theta}} \\
& \dot{\hat{\theta}}^{\mathrm{T}}=\Gamma_{\tilde{\theta}}^{-1}\left(\frac{\partial V_{\tilde{u}}}{\partial \tilde{u}}+\eta^{\mathrm{T}} \Gamma_{\eta}\right) f_{u \theta}\left(t, x, u, u_{c m d}\right) \\
& +\Gamma_{\tilde{\theta}}^{-1}\left(\frac{\partial L_{\hat{\theta}}^{\mathrm{T}}}{\partial u_{d}} \frac{\partial^{2} L_{\hat{\theta}}}{\partial \tilde{u} \partial u_{d}}+\frac{\partial L_{\hat{\theta}}^{\mathrm{T}}}{\partial \lambda} \frac{\partial^{2} L_{\hat{\theta}}}{\partial \tilde{u} \partial \lambda}\right) f_{u \theta}\left(t, x, u, u_{c m d}\right) \\
& +\Gamma_{\tilde{\theta}}^{-} \frac{\partial L_{\hat{\theta}}^{\mathrm{T}}}{\partial \lambda} \frac{\partial^{2} L_{\hat{\theta}}}{\partial x \partial \lambda} g(t, x) \Phi_{\theta}(t, x, u) \\
& +\Gamma_{\tilde{\theta}}^{-1} \frac{\partial L_{\hat{\theta}}^{\mathrm{T}}}{\partial u_{d}} \frac{\partial^{2} L_{\hat{\theta}}}{\partial x \partial u_{d}} g(t, x) \Phi_{\theta}(t, x, u)
\end{aligned}
$$

where $\mathbb{H}_{\hat{\theta}}:=\left(\begin{array}{cc}\frac{\partial^{2} L_{\hat{\theta}}}{\partial u_{d}^{2}} & \frac{\partial^{2} L_{\hat{\theta}}}{\partial \lambda \partial u_{d}} \\ \frac{\partial^{2} L_{\hat{\theta}}}{\partial u_{d} \partial \lambda} & 0\end{array}\right), \Gamma$ is a possibly time-varying symmetric positive definite weighting matrix, and $u_{f f \hat{\theta}}:=\mathbb{H}_{\hat{\theta}}^{-1} u_{F}$ is a feed-forward like

$u_{F}:=\left(\frac{\partial^{2} L_{\hat{\theta}}}{\partial t \partial u_{d}}, \frac{\partial^{2} L_{\hat{\theta}}{ }^{\mathrm{T}}}{\partial t \partial \lambda}\right)^{\mathrm{T}}+\left(\frac{\partial^{2} L_{\hat{\theta}}}{\partial x \partial u_{d}}, \frac{\partial^{2} L_{\hat{\theta}}{ }^{\mathrm{T}}}{\partial x \partial \lambda}\right)^{\mathrm{T}} f(t, x)$

$+\left(\frac{\partial^{2} L_{\hat{\theta}}^{\mathrm{T}}}{\partial x \partial u_{d}} \frac{\partial^{2} L_{\hat{\theta}}^{\mathrm{T}}}{\partial x \partial \lambda}\right)^{\mathrm{T}} g(t, x)\left(k_{x}(t, x)-\Phi\left(t, x, u_{d}+\tilde{u}, \hat{\theta}\right)\right)$

$+\left(\frac{\partial^{2} L_{\hat{\theta}}^{\mathrm{T}}}{\partial \tilde{u} \partial u_{d}} \frac{\partial^{2} L_{\hat{\theta}}^{\mathrm{T}}}{\partial \tilde{u} \partial \lambda}\right)_{\tilde{u}}^{\mathrm{T}}\left(t, x, \tilde{u}, u_{d}, \hat{\theta}, \hat{\theta}\right)+\left(\frac{\partial^{2} L_{\hat{\theta}}^{\mathrm{T}}}{\partial \hat{\theta} \partial u_{d}} \frac{\partial^{2} L_{\hat{\theta}}^{\mathrm{T}}}{\partial \hat{\theta} \partial \lambda}\right)^{\mathrm{T}} \hat{\hat{\theta}}^{\mathrm{T}}$

if $\operatorname{det}(\mathbb{H}) \neq 0$ and $u_{f f \hat{\theta}}:=0$ if $\operatorname{det}(\mathbb{H})=0$. Hence the time derivative of $V_{u_{d} \lambda \tilde{u} \eta \tilde{\theta}}$ along the trajectories of (1), (3), (6), (19) and (20) is:

$$
\begin{aligned}
& V_{u_{d} \lambda \tilde{u} \eta \tilde{\theta}}=-\eta^{\mathrm{T}} \Gamma_{\eta} A \eta-\alpha_{\tilde{u} 3}(|\tilde{u}|) \\
& -\left(\frac{\partial L^{\mathrm{T}}}{\partial u_{d}}, \frac{\partial L^{\mathrm{T}}}{\partial \lambda}\right) \mathbb{H}_{\hat{\theta}} \Gamma \mathbb{H}_{\hat{\theta}}\left(\frac{\partial L^{\mathrm{T}}}{\partial u_{d}}, \frac{\partial L^{\mathrm{T}}}{\partial \lambda}\right)^{\mathrm{T}} .
\end{aligned}
$$


In order to prove a global result we need to make an additional assumption on $\Phi_{\theta}(t, x, u)$. The assumption is based on the following lemma

Lemma 1. By Assumption 1 and (Mazenc and Praly 1996)'s lemma B.1 there exists continuous functions $\varsigma_{x}, \varsigma_{x u}, \varsigma_{u}: \mathbb{R}_{\geq 0} \rightarrow \mathbb{R}_{\geq 0}$, such that

$$
\begin{aligned}
\left|\Phi_{\theta}(t, x, u)\right| & \leq \varsigma_{x}(|x|) \varsigma_{x u}(|x|) \\
& +\varsigma_{x}(|x|) \varsigma_{u}\left(\left|z_{u_{d} \lambda \tilde{u} \tilde{\theta}}\right|_{\mathcal{O}_{u_{d} \lambda \tilde{u} \tilde{\theta}}}\right)
\end{aligned}
$$

where

$$
\begin{aligned}
\mathcal{O}_{u_{d} \lambda \tilde{\theta}}(t, x): & =\mathcal{O}_{u_{d} \lambda}(t, x) \\
& \times\left\{\left(\tilde{u}^{\mathrm{T}}, \eta^{\mathrm{T}}, \tilde{\theta}^{\mathrm{T}}\right) \in \mathbb{R}^{2 r+m} \mid\left(\tilde{u}^{\mathrm{T}}, \eta^{\mathrm{T}}, \tilde{\theta}^{\mathrm{T}}\right)=0\right\}
\end{aligned}
$$

and $z_{u_{d} \lambda \tilde{\theta}}:=\left(u_{d}^{\mathrm{T}}, \lambda^{\mathrm{T}}, \tilde{u}^{\mathrm{T}}, \eta^{\mathrm{T}}, \tilde{\theta}^{\mathrm{T}}\right)^{\mathrm{T}}$.

Assumption 2. (Continued)

c) There exists a $\mathcal{K}_{\infty}$ function $\alpha_{k}: \mathbb{R}_{\geq 0} \rightarrow \mathbb{R}_{\geq 0}$, such that

$$
\begin{gathered}
\alpha_{k}^{-1}(|x|) \alpha_{x 3}(|x|) \geq \alpha_{x 4}(|x|) \varsigma_{x \max }(|x|), \quad(22) \\
\text { where } \varsigma_{x \max }(|x|):=\max \left(1, \varsigma_{x}(|x|), \varsigma_{x}(|x|) \varsigma_{x u}(|x|)\right) .
\end{gathered}
$$

Proposition 1. If the assumptions 1, 2 and 3 are satisfied, then the solution of the closed-loop (16)(17) is bounded with respect to a set $\mathcal{O}_{x u_{d} \lambda \tilde{\theta}}(t):=$ $\mathcal{O}_{u_{d} \lambda \tilde{\theta}}(t, 0) \times\left\{x \in \mathbb{R}^{n} \mid x=0\right\}$. Furthermore $\mathcal{O}_{x u_{d} \lambda \tilde{\theta}}(t)$ is UGS with respect to the system (16)-(17). If in addition $\bar{f}_{u \theta}(t):=f_{u \theta}\left(t, x(t), u(t), u_{c m d}(t)\right)$ is Persistently Exited (PE), then $\mathcal{O}_{x u_{d} \lambda \tilde{\theta}}(t)$ is UGAS with respect to system (16)-(17).

PROOF. Sketch: The main steps of this proof is to prove: i) that (18) is a Lyapunov function, when $x(t)$ is assumed to be a signal that exists for all $t$, with respect to $\mathcal{O}_{u_{d} \lambda \tilde{\theta}}$, ii) boundedness and stability of the closed loop system and iii) attractivity when $\bar{f}_{u \theta}(t)$ is PE.

i) By similar arguments as in the proof of Proposition 1 (i) in (Tjønnås and Johansen 2005) it can be shown that $\mathcal{O}_{u_{d} \lambda \tilde{\theta}}$ is a closed forward invariant set with respect to $\Sigma_{2}$. Further it can be shown that system $\Sigma_{2}$ is finite escape time detectable through $\left|z_{u_{d} \lambda \tilde{\theta}}\right|_{\mathcal{O}_{u_{d} \lambda \tilde{\theta}}}$ and by using theorem 2.4.7 in (Abrahamson et al. 1988) and a similar approach as presented in the proof of Claim 3 in (Tjønnås and Johansen 2005), it can be shown that $V_{u_{d} \lambda \tilde{u} \eta \tilde{\theta}}\left(t, x, u_{d}, \lambda, \tilde{u}, \eta\right)$ is radially unbounded Lyapunov function with respect to $\left|z_{u_{d} \lambda \tilde{\theta}}\right|_{\mathcal{O}_{u_{d} \lambda \tilde{\theta}}}$

ii) By defining $v(t, x):=V_{x}(t, x)$, it can be shown from Assumption $2 \mathrm{c}$ ) and $V_{u_{d} \lambda \tilde{u} \eta \tilde{\theta}}$ that $|x(t)| \leq$ $M(r)$. Then by applying the cascade lemma from (Tjønnås et al. 2006) the UGS result is proved.

iii) By following ideas from the proof of the main result in (Tjønnås and Johansen 2005), the integral of $\tilde{\theta}^{\mathrm{T}} \tilde{\theta}$ can be shown to be bounded by the PE assumption, furthermore from this bound the uniform attractivity of the $\operatorname{set} \mathcal{O}_{x u_{d} \lambda \tilde{\theta}}$ can be shown by contradiction.
Proposition 1 implies that the time-varying first order optimal set $\mathcal{O}_{x u_{d} \lambda \tilde{\theta}}(t)$ is uniformly stable, and in addition uniformly attractive if a $\mathrm{PE}$ assumption is satisfied. Thus adaptive optimal control allocation is achieved asymptotically for the closed loop system (16)-(17).

Remark 2. If the unknown parameter vector $\theta$ in the effector model (2), is not the same as in the unknown parameter vector in the actuator dynamic (3), the low level adaptive control law defined in this paper may be combined with the adaptive law presented in (Tjønnås and Johansen 2005). The analysis of this case will be considered in future work.

\section{EXAMPLE}

In this section simulation results of an over-actuated scaled-model ship, manoeuvred at low-speed, is presented. The scale model-ship is moved while experiencing static disturbances, caused by wind and current, and propellers trust losses. The propeller losses can be due to: Axial Water Inflow, Cross Coupling Drag, Thruster-Hull and Thruster-Thruster Interaction (see (Sørensen et al. 1997) and (Fossen and Blanke 2000) for details). But in this example we limit our study to thruster loss caused by Thruster-Hull interaction. A 3DOF horizontal plane model described by:

$$
\begin{aligned}
\dot{\eta}_{e} & =R\left(\psi_{p}\right) \nu \\
\dot{\nu} & =-M^{-1} D \nu+M^{-1}(\tau+b) \\
\tau & =\Phi(u, \theta),
\end{aligned}
$$

is considered, where $\eta_{e}:=\left(x_{e}, y_{e}, \psi_{e}\right)^{\mathrm{T}}:=\left(x_{p}-\right.$ $\left.x_{d}, y_{p}-y_{d}, \psi_{p}-\psi_{d}\right)^{\mathrm{T}}$ is the north and east positions and compass heading deviations. Subscript $p$ and $d$ denotes the actual and desired states. $\nu:=\left(v_{x}, v_{y}, r\right)^{\mathrm{T}}$ is the body-fixed velocities in surge, sway and yaw, $\tau$ is the generalized force vector, $b:=\left(b_{1}, b_{2}, b_{3}\right)^{\mathrm{T}}$ is a static disturbance and $R\left(\psi_{p}\right)$ is the rotation matrix function between the body fixed and the earth fixed coordinate frame. The example we present here is based on (Lindegaard and Fossen 2003), and is also studied in (Johansen 2004) and (Tjønnås and Johansen 2005). In the considered model there are five force producing devices; the two main propellers aft of the hull, in conjunction with two rudders, and one tunnel thruster going through the hull of the vessel. $\omega_{i}$ denotes the propeller angular velocity and $\delta_{i}$ denotes the rudder deflection. $i=1,2$ denotes the aft actuators, while $i=3$ denotes the tunnel thruster. Eq. (23) can be rewritten in the form of (1) and (2) by:

$$
\begin{gathered}
x:=\left(\eta_{e}, \nu\right)^{\mathrm{T}}, \theta:=\left(\theta_{1}, \theta_{2}, \theta_{3}\right)^{\mathrm{T}}, \tau:=\left(\tau_{1}, \tau_{2}, \tau_{3}\right)^{\mathrm{T}} \\
u:=\left(\omega_{1}, \omega_{2}, \omega_{3}, \delta_{1}, \delta_{2}\right)^{\mathrm{T}}, f_{x}:=\left(\begin{array}{c}
R\left(\psi_{e}+\psi_{d}\right) \nu \\
-M^{-1} D \nu+M^{-1} b
\end{array}\right), \\
\Phi(\nu, u, \theta):=G_{u}(u)\left(\begin{array}{c}
T_{1}\left(v_{x}, \omega_{1}, \theta_{1}\right) \\
T_{2}\left(v_{x}, \omega_{2}, \theta_{2}\right) \\
T_{3}\left(v_{x}, v_{y}, \omega_{3}, \theta_{3}\right)
\end{array}\right), g_{x}:=\left(\begin{array}{c}
0 \\
M^{-1}
\end{array}\right), \\
G_{u}(u):=\left(\begin{array}{ccc}
\left(1-D_{1}\right) & \left(1-D_{2}\right) & 0 \\
L_{1} & L_{2} & 1 \\
\Phi_{\theta 31} & \Phi_{\theta 32} & l_{3, x}
\end{array}\right) \\
\Phi_{3 i}(u):=-l_{i, y}\left(1-D_{i}(u)+l_{i, x} L_{i}(u)\right) .
\end{gathered}
$$

The truster forces are given by: 


$$
\begin{gathered}
T_{i}\left(v_{x}, \omega_{i}, \theta_{i}\right):=T_{n i}\left(\omega_{i}\right)-\phi_{i}\left(\omega_{i}, v_{x}\right) \theta_{i} \\
T_{n i}\left(\omega_{i}\right):=\left\{\begin{array}{cc}
k_{T p_{i}} \omega_{i}^{2} & \omega_{i} \geq 0 \\
k_{T n_{i}}\left|\omega_{i}\right| \omega_{i} & \omega_{i}<0
\end{array},\right. \\
\phi_{i}\left(\omega_{i}, v_{x}\right)=\omega_{i} v_{x}, \quad \phi_{3}\left(\omega_{3}\right):=\sqrt{\left(v_{x}^{2}+v_{y}^{2}\right)}\left|\omega_{3}\right| \omega_{3} \\
\theta_{i}=\left\{\begin{array}{cc}
k_{T \theta_{i}}(1-w) & v_{x} \geq 0 \\
k_{T \theta_{i}} & v_{x}<0
\end{array}, \theta_{3}:=k_{T \theta_{3}}\right.
\end{gathered}
$$

where $0<w<1$ is the wake fraction number, $\phi_{i}\left(\omega_{i}, v_{x}\right) \theta_{i}$ is the thrust loss due to changes in the advance speed, $v_{a}=(1-w) v_{x}$, and the unknown parameters $\theta_{i}$ represents the thruster loss factors dependent on whether the hull invokes on the inflow of the propeller or not. The rudder lift and drag forces are projected through:

$L_{i}(u):= \begin{cases}\left(1+k_{L n_{i}} \omega_{i}\right)\left(k_{L \delta 1_{i}}+k_{L \delta 2_{i}}\left|\delta_{i}\right|\right) \delta_{i}, & \omega_{i} \geq 0 \\ 0 & , \omega_{i}<0\end{cases}$
$D_{i}(u):=\left\{\begin{array}{ll}\left(1+k_{D n_{i}} \omega_{i}\right)\left(k_{D \delta 1_{i}}\left|\delta_{i}\right|+k_{D \delta 2_{i}} \delta_{i}^{2}\right), & \omega_{i} \geq 0 \\ 0 & , \omega_{i}<0\end{array}\right.$.

Furthermore it is clear from (24) that $\Phi(\nu, u, \theta)=$ $G_{u}(u) Q(u)+G_{u}(u) \phi\left(\omega, v_{x}\right) \theta$, where $\phi\left(\omega, v_{x}\right):=$ $\operatorname{diag}\left(\phi_{1}, \phi_{2}, \phi_{3}\right)$ and $Q(u)$ represents the nominal propeller thrust.

The actuator error dynamic for each propeller is based on the propeller model presented in (Pivano et al. 2007) and given by

$$
\begin{gathered}
J_{m i} \dot{\tilde{\omega}}_{i}=-k_{f i}\left(\tilde{\omega}_{i}+\omega_{d i}\right)-\frac{T_{n i}}{a_{T}}\left(\tilde{\omega}_{i}+\omega_{d i}\right) \\
+\frac{\phi_{i}\left(\omega_{i}, v_{x}\right) \theta_{i}}{a_{T}}+u_{c m d i}-J_{m i} \dot{\omega}_{d i}
\end{gathered}
$$

where $\tilde{\omega}_{i}:=\left(\omega_{i}-\omega_{i d}\right), J_{m}$ is the shaft moment of inertia, $k_{f}$ is a positive coefficient related to the viscous friction, $a_{T}$ is a positive model constant (Pivano et al. 2006) and $u_{c m d}$ is the motor torque. By the quadratic Lyapunov function $V_{\tilde{\omega}_{i}}:=\frac{\tilde{\omega}_{i}^{2}}{2}$ it is easy to see that the control law

$$
\begin{aligned}
u_{c m d i} & :=-K_{\omega p} \tilde{\omega}_{i}-\frac{\phi_{i}\left(\omega_{i}, v_{x}\right) \hat{\theta}_{i}}{a_{T}}+J_{m i} \dot{\omega}_{d i} \\
& +\frac{T_{n i}\left(\omega_{d i}\right)}{a_{T}}+k_{f i} \omega_{d i} .
\end{aligned}
$$

makes the origin of (25) UGES when $\hat{\theta}_{i}=\theta_{i}$. The error dynmaics of the rudder is given by:

$$
m_{i} \dot{\tilde{\delta}}=a_{i}\left(\tilde{\delta}+\delta_{d i}\right)+b_{i} u_{c m d \delta i}-m_{i} \dot{\delta}_{d i}
$$

where $\tilde{\delta}:=\delta_{i}-\delta_{d i}, a_{i}(t)$ is a known scalar function, $b_{i}$ is a known scalar parameter bounded away from zero, and the controller

$b_{i} u_{c m d \delta i}:=-K_{\delta} \tilde{\delta}-a_{i}(t)\left(\tilde{\delta}+\delta_{d i}\right)+m_{i} \dot{\delta}_{d i}$ (28) makes the origin of (27) UGES. The parameters for the actuator model and controllers are: $a_{T}=1$, $J_{m i}=10^{-2}, k f_{i}=10^{-4}, a_{i}=-10^{-4}, b_{i}=10^{-5}$, $m_{i}=10^{-2}, K_{\omega p}=5 \cdot 10^{-3}$ and $K_{\delta}=10^{-3}$

A virtual controller $\tau_{c}$ that stabilizes the system (23) uniformly, globally and exponentially, for some physically limited yaw rate, is proposed in (Lindegaard and Fossen 2003) and given by

$$
\tau_{x}:=-K_{i} R^{T}\left(\psi_{p}\right) \xi-K_{p} R^{T}\left(\psi_{p}\right) \eta_{e}-K_{d} \nu,
$$
where (23) is augmented with the integral action state, $\dot{\xi}=\eta_{e}$.

The cost function designed for the optimization problem, (4), is:
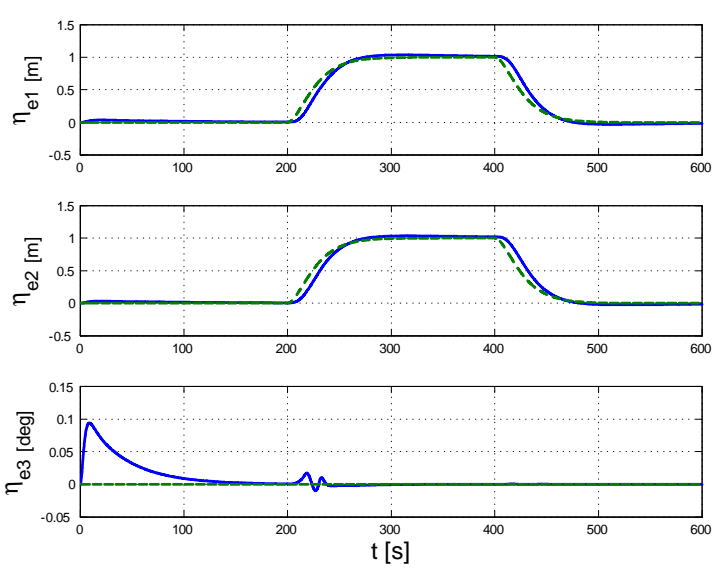

Fig. 2. Desired (dashed) and actual ship positions (solid).
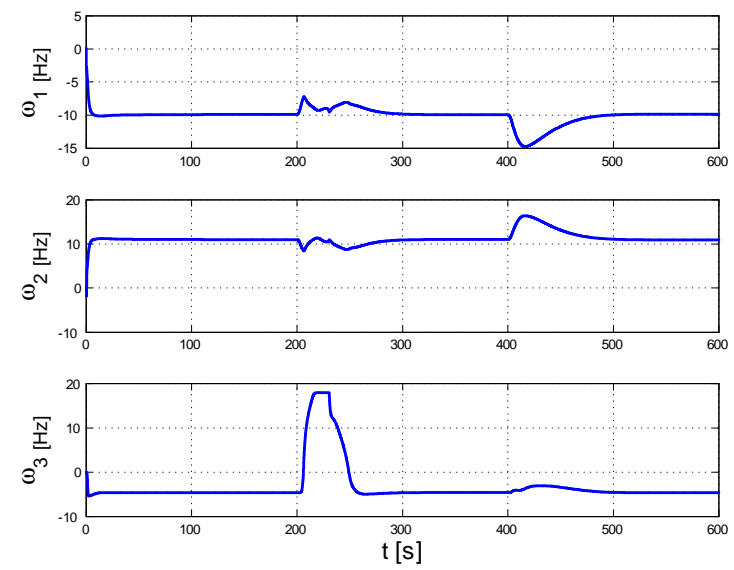

Fig. 3. Actual propeller velocities

$$
\begin{aligned}
& J(u):=\sum_{i=1}^{3} k_{i}\left|\omega_{i}\right| \omega_{i}^{2}+\sum_{i=1}^{2} q_{i} \delta_{i}^{2}-\varsigma \sum_{i=1}^{3} \lg \left(-\omega_{i}+18\right) \\
& -\varsigma \sum_{i=1}^{3} \lg \left(\omega_{i}+18\right)-\varsigma \sum_{i=1}^{2} \lg \left(-\delta_{i}+35\right)-\varsigma \sum_{i=1}^{2} \lg \left(\delta_{i}+35\right), \\
& w_{\delta}=0.05, k_{1}=k_{2}=0.01, k_{3}=0.02, q_{1}=q_{2}=500 .
\end{aligned}
$$

The gain matrices are chosen as follows: $A_{\hat{u}}:=$ $2 I_{5 \times 5}, \Gamma_{\tilde{\theta}}:=10^{-3}, \Gamma_{\eta}:=\operatorname{diag}\left(10^{3}, 10^{3}, 3\right)$ and $\Gamma:=\left(\mathbb{H}_{\hat{\theta}}^{\mathrm{T}} W \mathbb{H}_{\hat{\theta}}+\varepsilon I\right)^{-1}$ where

$W:=\operatorname{diag}(1,1,1,1,1,0.9,0.9,0.7)$ and $\varepsilon:=10^{-9}$. The weighting matrix $W$ is defined such that the deviation of $\left|\frac{\partial L_{\hat{\theta}}}{\partial \lambda}\right|=|k(t, x)-\Phi(t, x, u, \hat{\theta})|$ from zero is penalized more then the deviation of $\left|\frac{\partial L_{\hat{\theta}}}{\partial u}\right|$ from zero in the search direction.

The static disturbance vector is $b:=0.05(1,1,1)^{\mathrm{T}}$, and the thruster loss vector $\theta$ is given in Figure 6.

The simulation results are presented in the Figures 2-7. The control objective is satisfied and the commanded virtual controls are tracked closely by the forces generated by the adaptive control allocation law: see Figure 5. Note that there are some deviations since $\omega$ saturates at ca. $220 \mathrm{~s}$ and $420 \mathrm{~s}$. Also note that the parameter estimates only converge to the true values when the ship is moving and the thrust loss is not zero. The simulations are carried out in a discrete MATLAB environment with a sampling rate of $20 \mathrm{~Hz}$. 

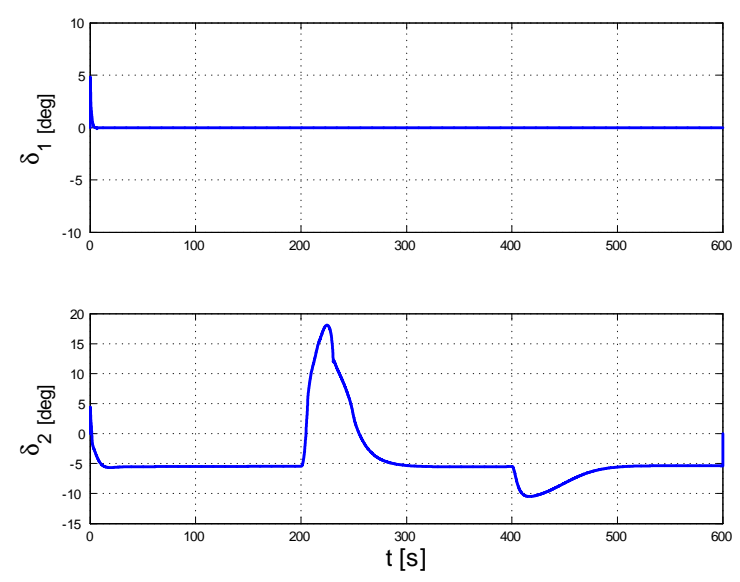

Fig. 4. Actual rudder deflection
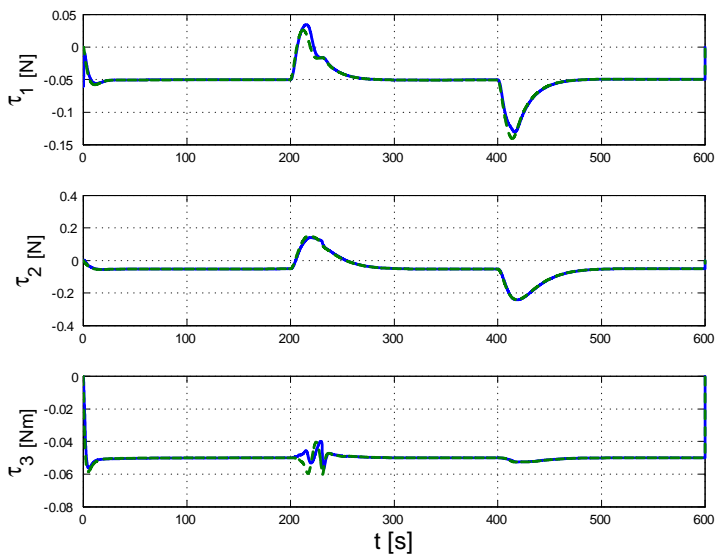

Fig. 5. The virtual control (dashed) and actual (solid)
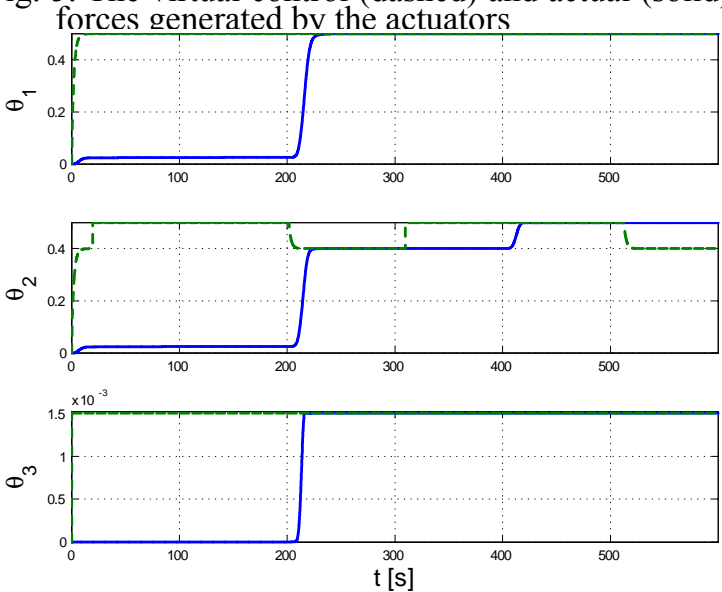

Fig. 6. Actual (dashed) and estimated (solid) loss parameters

\section{ACKNOWLEDGEMENT}

The authors are grateful to Luca Pivano at NTNU, for insightful comments. This work is sponsored by the Research Council of Norway through the Strategic University Programme on Computational Methods in Nonlinear Motion Control

\section{REFERENCES}

Abrahamson, H., J. E. Marsden and T. Ratiu (1988). Manifolds, Tensor Analysis and applications. 2'nd ed.. Springer-Verlag.

Bodson, M. (2002). Evaluation of optimization methods for control allocation. J. Guidance, Control and Dynamics 25, 703-711.
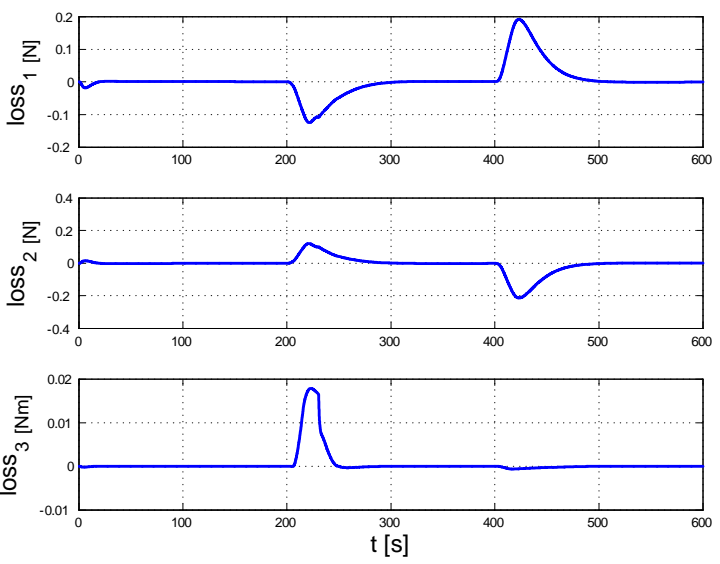

Fig. 7. Actual thrust loss

Enns, D. (1998). Control allocation approaches. In: Proc. AIAA Guidance, Navigation and Control Conference and Exhibit, Boston MA. pp. 98-108.

Fossen, T. I. and M. Blanke (2000). Nonlinear output feedback control of underwater vehicle propellers using feedback form estimated axial flow velocity. IEEE JOURNAL OF OCEANIC ENGINEERING 25(2), 241-255.

Johansen, T. A. (2004). Optimizing nonlinear control allocation. Proc. IEEE Conf. Decision and Control. Bahamas pp. 34353440.

Johansen, T. A., T. I. Fossen and Svein P. Berge (2004). Constrained nonlinear control allocation with singularity avoidance using sequential quadratic programming. IEEE Trans. Control Systems Technology 12, 211-216.

Lin, Y., E. D. Sontag and Y. Wang (1996). A smooth converse lyapunov theorem for robust stability. SIAM Journal on Control and Optimization 34, 124-160.

Lindegaard, K. P. and T. I. Fossen (2003). Fuel-efficient rudder and propeller control allocation for marine craft: Experiments with a model ship. IEEE Trans. Control Systems Technology 11, 850-862.

Luo, Y., A. Serrani, S. Yurkovich, D.B. Doman and M.W. Oppenheimer (2004). Model predictive dynamic control allocation with actuator dynamics. In Proceedings of the 2004 American Control Conference, Boston, MA.

Luo, Y., A. Serrani, S. Yurkovich, D.B. Doman and M.W. Oppenheimer (2005). Dynamic control allocation with asymptotic tracking of time-varying control trajectories. In Proceedings of the 2005 American Control Conference, Portland, OR.

Mazenc, F. and L. Praly (1996). Adding integrations, saturated controls, and stabilization for feedforward systems. IEEE Transactions on Automatic Control 41, 1559-1578.

Pivano, L., Ø. N. Smogeli, T. A. Johansen and T. I. Fossen (2006). Marine propeller thrust estimation in four-quadrant operations. 45th IEEE Conference on Decision and Control, San Diego, CA, USA.

Pivano, L., T. A. Johansen, Ø. N. Smogeli and T. I. Fossen (2007). Nonlinear Thrust Controller for Marine Propellers in FourQuadrant Operations. American Control Conference (ACC), New York, USA.

Poonamallee, V., S. Yurkovich, A. Serrani, D.B. Doman and M.W. Oppenheimer (2005). Dynamic control allocation with asymptotic tracking of time-varying control trajectories. In Proceedings of the 2004 American Control Conference, Boston, $M A$.

Sørdalen, O. J. (1997). Optimal thrust allocation for marine vessels. Control Engineering Practice 5, 1223-1231.

Sørensen, A. J., A. K. Ådnanes, T. I. Fossen and J. P. Strand (1997). A new method of thruster control in positioning of ships based on power control. Proc. 4th IFAC Conf. Manoeuvering and Control of Marine Craft, Brijuni, Croatia, .

Teel, A., E. Panteley and A. Loria (2002). Integral characterization of uniform asymptotic and exponential stability with applications. Maths. Control Signals and Systems 15, 177-201.

Tjønnås, J., A. Chaillet, E. Panteley and T. A. Johansen (2006). Cascade lemma for set-stabile systems. 45th IEEE Conference on Decision and Control, San Diego, CA.

Tjønnås, J. and T. A. Johansen (2005). Adaptive optimizing nonlinear control allocation. In Proc. of the 16th IFAC World Congress, Prague, Czech Republic. 